\title{
La conformación de valores noticia en un contexto de conflicto. Análisis pragmático de su funciona- miento en la información sobre el narcotráfico en México y el feminicidio de Ciudad Juárez
}

\author{
Berta GARCÍA Orosa \\ Universidad de Santiago de Compostela \\ bertago@gmail.com \\ Santiago GaLlur SANTORUM \\ Universidad Autónoma de Ciudad Juárez (México) \\ santiagostgs@hotmail.com
}

Recibido: 16 de julio de 2012

Aceptado: 28 de noviembre de 2012

\begin{abstract}
Resumen
El artículo reflexiona sobre la utilización de los valores noticia tradicionales en una situación de conflicto concreta: la información sobre el feminicidio de Ciudad Juárez y sobre el narcotráfico en Mexico. Para ello se estudian los tres medios de comunicación con una mayor cobertura de ambos temas en todo el país desde 1976 hasta la actualidad a través de la triangulación metodológica (fuentes bibliográficas, entrevistas y análisis de contenido). A lo largo de la investigación se detecta la importancia del contexto informativo en la producción informativa y se comprueba una dislocación del empleo de los valores noticia tradicionales al mismo tiempo que se desvelan seis factores de noticiabilidad que permiten la visibilización de una gran cantidad de aspectos concretos sobre el feminicidio en Ciudad Juárez y sobre el narcotráfico en todo el país, que son obviados por los principales medios de comunicación generalistas mexicanos.
\end{abstract}

Palabras clave: noticiabilidad, conflicto, periodismo.

The news value in a context of conflict. Pragmatic analysis of their operation in the information about drugs trade in Mexico and femicide in Ciudad Juárez

\begin{abstract}
This article ponders over the use of the traditional news value in a very specific situation of conflict: the information about the femicide in Ciudad Juárez and the drugs trade in México. Therefore, the three media in the whole country with the biggest coverage of both subjects since 1976 till nowadays have been studied through a methodological triangular structure (bibliographie sources, interviews and content analysis). Along this research it is noticed the importance of the informative context in the news production and it is ascertained a dislocation using the traditional news value therewithal are revealed six newsworthiness factors the enable the visibility of a great amount of specific aspects about the femicide in Ciudad Juárez and about the drugs trade all around the country, that are concealed by the main mexican generalistic media.
\end{abstract}

Keywords: newsworthiness, conflict, journalism.

Referencia normalizada: GARCÍA OROSA, Berta y GALLUR SANTORUM, Santiago (2012): "La conformación de valores noticia en un contexto de conflicto. Análisis pragmático de su funcionamiento en la información sobre el narcotráfico en México y el feminicidio de Ciudad Juárez". Estudios sobre el mensaje periodístico, vol. 18, núm. 2 (julio-diciembre), págs.: 491-511. Madrid, Servicio de Publicaciones de la Universidad Complutense.

Sumario: 1. Introducción. 2. Los valores noticia como filtro de la información. 3. Metodología de la investigación. 4. Análisis de datos. 5. Conclusiones. 6. Referencias bibliográficas. 7. Anexos; 7.1. Anexo 
I. Entrevistas realizadas; 7.2. Anexo II. Informaciones relevantes; 7.3. Anexo III. Extractos de noticias con la transformación de los valores noticia.

\section{Introducción}

Los valores noticia constituyen uno de los filtros entre los acontecimientos y la realidad publicada por los medios de comunicación pero, lejos de ser factores absolutos y perpetuos, están sometidos al contexto comunicativo y afectados por la pragmática informativa. Las situaciones de conflicto constituyen un contexto complejo de confrontación entre las diferentes partes que influyen especialmente en la conformación de estos criterios o barreras de noticiabilidad. Aún así, cada situación de conflicto presenta peculiaridades que influyen especialmente en la construcción de la realidad por parte de los medios de comunicación especialmente en aquellos casos en los que los periodistas no sólo actúan como meros transmisores de los acontecimientos sino que, además de narradores y comentaristas, son actores del conflicto por iniciativa propia en algunas ocasiones y simplemente por reacción en otras (BORRAT, 1989: 16). El artículo nace de la reflexión sobre la reconfiguración pragmática de los valores noticias en un caso de conflicto que, desgraciadamente, cada día adquiere mayor visibilidad internacional: la información sobre las desapariciones de mujeres en Ciudad Juárez y el narcotráfico en México. En las próximas líneas analizaremos y trataremos de establecer los valores noticia creados en este conflicto en el que, además de diferentes actores sociales, los periodistas actúan, en muchas ocasiones, como parte implicada.

\section{Los valores noticia como filtro de la información}

Los valores noticia han sido utilizados desde la preceptiva periodística como uno de los principales filtros que debe superar un hecho para convertirse en noticia fundamentalmente desde los años 60 del pasado siglo. Pese al surgimiento de algunas voces críticas y algunas modificaciones en su empleo como la inclusión de nuevos factores provenientes del conocimiento psicológico, los factores íntimos y personales del autor de la información y la ampliación de su uso a los procesos de selección que hacen las audiencias (EILDERS, 2006: 9) y a la importancia del contexto comunicativo en el que se desarrollen, siguen siendo válidos para la realización de las noticias y la comprensión de los mecanismos de construcción de la realidad en los análisis del discurso.

Como decíamos, se estudian fundamentalmente en los años 60 y 70 con la emergencia de las tradiciones escandinava y alemana de investigación sobre el tema, a la que hay que añadir la estadounidense, y tiene otro repunte en los 90 del siglo XX. Sin embargo, pueden encontrarse ya algunas investigaciones a principios de los años veinte. Es en esta época cuando Lippmann introduce las ideas de sensacionalismo, proximidad, relevancia, desambiguación y facticidad como atributos que aportaban valor como noticia a los sucesos.

Cuando Walter Lippman introdujo el concepto de valor noticia trataba de explicar por qué los periodistas cubrían algunos sucesos y pasaban por alto otros (KEPPLINGER y EHMIG, 2006: 25). Según él los acontecimientos eran noticia porque estaban caracterizados por configuraciones que más tarde se llamarían factores noticia que los convertían en valiosos. Por ejemplo, si la historia implicaba a instituciones influyentes o 
si incrementaba el daño o la ruptura del orden tenían más posibilidades de convertirse en noticia.

A lo largo de la teoría de la redacción periodística se intentaron sistematizar distintos mecanismos o dispositivos mentales que activa el periodista para decidir qué acontecimientos son noticia y cuáles no, los valores noticia, y se dieron diferentes soluciones. Fueron muchos los autores que elaboraron listados de factores, características o peculiaridades de los hechos y/o temas que potencian su conversión en noticia.

La trayectoria de los trabajos publicados en lengua castellana, fundamentalmente en España y en México, es similar. Según indica SerRANo (2003, p.161) es clásica la cita de Ismael HerRáiz, en el texto de Nicolás GonzÁlez Ruiz, en la que afirma que en las escuelas de Periodismo españolas se estudia la lista de los caracteres de la noticia o, con mayor precisión, de los factores objetivos de interés público, de las circunstancias que pueden actuar sobre un acontecimiento convirtiéndolo en noticia. $\mathrm{Ni}$ que decir tiene que estas relaciones demuestran ciertas variedades de criterio y que al incorporarse a nuestros manuales y tratados, han sufrido modificaciones y ampliaciones. He aquí respectivamente dos listas de composición norteamericana y una de confección española: 1) proximidad temporal, proximidad física, consecuencia, prominencia, drama, atracción, conflicto, sexo, emoción y progreso; 2) actualidad, proximidad, prominencia, importancia, rareza, interés humano, amor a la lucha, emociones, deseos de superación y entretenimiento; 3) actualidad, proximidad y familiaridad, eminencia o celebridad, novedad o rareza, vida, rivalidad y lucha, sentimientos, amor, utilidad, dinero o propiedad, diversiones y deportes, intereses locales y generales, intereses domésticos, moralidad, cultura y número y calidad de los lectores afectados (SERRANo, 2003). Asimismo, teóricos de la relevancia de Gonzalo Martín Vivaldi, Lorenzo Gomis, José Luis Martínez Albertos y Josep María Casasús partieron de la clasificación de Warren para la valoración de los criterios de selección de la información (CASALS, 2005, p. 195). A partir de estas primeras aportaciones diversos investigadores actualizaron y adaptaron a sus realidades los valores noticia.

Cada una de las propuestas teóricas se adapta y se reconfigura por una pragmática informativa que la activa y le otorga significado. En el caso analizado, la información sobre el feminicidio en Ciudad Juárez y el narcotráfico en México, el contexto alcanza una gran influencia sobre la producción de la información periodística e incluso a veces provoca la dislocación o incluso inversión de estos factores. Para su análisis elaboramos una clasificación propia de valores noticia que resume y adapta a la situación estudiada las propuestas teóricas desarrolladas en este apartado y que se ofrece en el próximo capítulo.

\section{Metodología de la investigación}

El objetivo principal de la investigación es analizar el comportamiento de los valores noticia en dos situacines conflictivas concretas: la desaparición de mujeres en Ciudad Juárez y el narcotráfico en México. Para ello estudiaremos el comportamiento de los valores noticia tradicionales en la información publicada sobre los temas y, a partir de ahí, elaboraremos una propuesta de factores de noticiabilidad que será posteriormente validada o refutada a través del análisis empírico. 
La hipótesis principal indica que en un contexto de violencia extrema constante en el que los periodistas han visto tremendamente limitado su trabajo, tres medios de comunicación mexicanos -Proceso, Contralínea y CIMAC- han utilizado una nueva forma de cobertura informativa consistente en la reconfiguración e incluso inversión de los valores noticia tradicionales en el tratamiento del narcotráfico y el feminicidio de Ciudad Juárez. Esta dislocación de la noticiabilidad permite explicar el conflicto como estructural y no como un simple conflicto coyuntural presionados por la necesidad de informar inmediatamente y provoca que las víctimas y aquellas personas "sin voz" en los medios tengan cabida, pudiendo aportar su propia versión de los hechos, contrastando o incluso sustituyendo a la versión oficial.

Para comprender esta realidad es necesario conocer las características de los medios de comunicación seleccionados para el estudio. Como indicábamos, se trata de dos revistas de periodismo de investigación por su cobertura exhaustiva del narcotráfico, Proceso y Contralínea, y de la agencia de noticia CIMAC (Comunicación e Información de la Mujer A.C) por su cobertura del feminicidio de Ciudad Juárez. Hemos escogido estos tres medios porque han conseguido hacer visibles hechos invisibilizados en casi todos los medios de comunicación mexicanos, debido a que el tipo de temática que cubren (narcotráfico y feminicidio) impide a sus noticias superar la barrera de los valores-noticia de las cabeceras tradicionales. Veamos brevemente sus características.

El primero de ellos es Proceso. Fundado en 1976 por Julio García Scherer después de la expulsión de varios periodistas del diario Excélsior justo al final del sexenio de Luís Echeverría, la revista semanal Proceso supuso en sus orígenes una alternativa informativa de la izquierda mexicana. Si bien hoy en día es uno de los medios más respetados en México por el tipo de temas que trata y los llamativos titulares que utiliza, en la actualidad se puede apreciar como la seriedad original de la información que proporciona ha dejado paso a cierto toque de sensacionalismo en algunas de sus noticias (no es así en las del tema analizado). Así, a pesar de que esta tendencia sólo se aprecia de forma constante en el tratamiento de temas políticos, dicha dinámica les ha provocado serios problemas derivados sobre todo de sus titulares y portadas, con fotografías en determinadas ocasiones agresivas y repletas de morbo. Es más, el tipo de información manejada y el hecho de que frecuentemente la atribución de las fuentes no es siempre directa en temas tan delicados como el narco, ha provocado una gran polémica. Concretamente uno de sus periodistas más respetados, Ricardo Ravelo ${ }^{1}$ (especialista en narcotráfico) fue acusado por la cadena de televisión

\footnotetext{
${ }^{1}$ A mediados de agosto del 2010, Santiago Gallur consiguió concertar después de muchos intentos una entrevista con Ricardo Ravelo, con el objetivo de aclarar algunos conceptos sobre las informaciones publicadas por éste sobre el narco en la revista Proceso. Después de intentarlo hasta en dos ocasiones en la sede central de Proceso en México D.F., a la hora convenida, el experto en narcotráfico no pudo llegar a la cita. Posteriormente, y como solución extraordinaria se propuso una entrevista telefónica debido a los compromisos profesionales de Ravelo. Así, se esperó durante más de tres horas esperando la llamada telefónica. Esta nunca se produjo.
} 
pública Televisa de recibir dinero del narco por no publicar determinadas informaciones. Y es que poco tiempo antes la revista publicaba una entrevista que su director realizó al narcotraficante más buscado de México en esos momentos, el Mayo Zambada, con una fotos en portada de ambos posando amistosamente para la cámara.

A pesar de todos estos cuestionamientos y agrias polémicas desatadas sobre el uso de sus fuentes, Proceso cuenta con la reputación de ser una de las revistas más serias del país, gracias a la gran calidad que siempre ha demostrado en sus noticias. Además es de las pocas que dedica una sección fija al narcotráfico, en donde se suelen mencionar los nombres de una gran cantidad de funcionarios, militares y políticos de alto nivel corruptos y que trabajan para el narco. Por ello, a pesar de la innegable necesidad de contrastar todos los detalles de las informaciones extraídas de esta revista, su consulta se ha situado por derecho propio como imprescindible en cualquier análisis académico del narco mexicano en la actualidad.

El segundo medio analizado es, Contralínea, bien distinto aunque con aspectos en común con el medio de Scherer. Así, surgida en 2002 y dirigida por Miguel Badillo, esta revista semanal ha aspirado desde un principio a ofrecer aquello que ya publicita en su nombre: periodismo de investigación. Por ello, su línea se mantiene constante a lo largo de estos 10 años de existencia: información crítica y de izquierdas. Podemos decir, que pretende cubrir el hueco que ha dejado Proceso con el paso de los años por su suave giro hacia el sensacionalismo. De esta forma, siempre sobria y con noticias contundentes alejadas de lo banal y cercanas a la ideología más de izquierdas compatible con el periodismo independiente, Zósimo Camacho, su coordinador de información asegura que nunca han publicado algo de lo que no tengan todas las pruebas que les permiten contrastar al cien por cien la información que publican. Prueba de ello, como afirma Camacho, es que nunca les han interpuesto ninguna demanda por sus informaciones publicadas, a pesar de la contundencia de las mismas ${ }^{2}$. Sin embargo, como la revista y la Comisión Nacional de Derechos Humanos han denunciado a lo largo de los últimos años, el gobierno federal, debido a la gran carga crítica de las informaciones publicadas por Contralínea, ha llevado a cabo una serie de acciones de intimidación, amenazas y presión económica sobre el medio para provocar su cierre o al menos el cese de este tipo de periodismo de investigación tremendamente hostil hacia el poder establecido.

A pesar de todo la revista sigue sacando a la luz todo tipo de tramas de corrupción, lavado de dinero y vínculo de los diferentes cárteles con la policía y el ejército mexicano e incluso de otros países como Guatemala. El problema es parecido al de Proceso, ya que en ocasiones, aunque aseguran que las fuentes están perfectamente contrastadas, no se realiza la atribución directa debida fundamentalmente a que la identificación completa de las fuentes en sus noticias sobre el narcotráfico podría poner en serio peligro la seguridad de las mismas, por la gran relevancia de los datos

2 Declaraciones extraídas de la entrevista realizada con Zósimo CAMACHO y Nancy FlORES a mediados de agosto del 2010, en la sede de la Revista Contralínea, en México D.F. 
publicados. De esta forma, se abre el debate interesado, abierto en muchas ocasiones por los políticos y el propio gobierno sobre si es válida la información que no tenga una atribución directa de la fuente, a pesar de que en su conjunto, contrastada con otras fuentes también de medios, encaje y resulte completamente veraz.

En una línea completamente distinta se sitúa $C I M A C$, Comunicación e Información de la Mujer, A. C., el tercer medio de comunicación analizado, que juega un papel importantísimo en la documentación periódica de todo lo relacionado con el feminicidio de Ciudad Juárez. Para entender la importancia de dicho medio es necesario remontarse a 1988, ya que es cuando surge un medio de comunicación nuevo y distinto a todos aquellos conocidos hasta el momento en México, CIMAC, Comunicación e Información de la Mujer, A.C.: “...es una institución multimedia que desde 1988 promueve en los medios de comunicación un nuevo punto de vista sobre la condición actual de las mujeres en México y el mundo, siempre vinculado con los principios de equidad, justicia social y democracia" tal y como indican en su web.

Así, dirigida en 2010 por Lucía Lagunes, la creación de esta agencia de comunicación resulta esencial en la presión social ejercida por las familias de las víctimas de los asesinatos y desapariciones de mujeres, ya que la cobertura continuada que hace del feminicidio se convierte en un factor clave para comprender la evolución de las distintas estrategias llevadas a cabo por el Gobierno mexicano en las investigaciones del feminicidio de Juárez. Y es que a partir de 1999, en vista de que los asesinatos y desapariciones de mujeres en Ciudad Juárez no cesaban, CIMAC decide hacer un seguimiento exhaustivo de todo lo relacionado con estos crímenes, prestando especial atención a las distintas acciones llevadas a cabo por las familias de las víctimas y sus asociaciones. Asimismo, este seguimiento permite difundir a nivel mediático la lucha que desde 1993 llevan a cabo las familias de las víctimas, consiguiendo convertirse a la vez en una plataforma desde donde las distintas organizaciones, asociaciones e investigadores que se interesan sobre el tema, pueden manifestar su discrepancia con respecto a las teorías y actitudes de las autoridades mexicanas. De esta forma, CIMAC puso a disposición de todo aquel interesado en el tema, de forma gratuita a través de Internet, un archivo hemerográfico constituido por más de 2000 noticias sobre distintos aspectos vinculados a los feminicidios de Juárez desde 1999 hasta la actualidad.

Lo característico de este medio es que identifica siempre de manera precisa todas las fuentes que utiliza para la elaboración de sus artículos, noticias y reportajes. Asimismo, invierte la faceta del periodismo habitualmente dando voz a las familias de las víctimas y a toda una serie de expertos y personalidades disidentes de las versiones oficiales del gobierno, señalando en una parte relevante la implicación del narcotráfico en parte de los asesinatos y desapariciones de mujeres en Juárez. De esta forma, $C I M A C$ ofrece a través de su web un acervo importantísimo de noticias sobre el seguimiento cronológico de todos los hechos relacionados con el feminicidio de Juárez, fundamentalmente compuesto por declaraciones de un gran espectro de personalidades vinculadas de uno u otro modo con las investigaciones llevadas a cabo sobre estos crímenes.

Para la investigación sobre estos tres medios se utilizó la triangulación metodológica y los siguientes instrumentos de investigación: 
1. Fuentes consultadas. A continuación se presenta un esquema orientativo de las fuentes utilizadas divididas por tipo y temática a tratar ${ }^{3}$ :

-Informes, clasificados por temática serían los siguientes: -Derechos Humanos en México; -Derechos Humanos en América Latina; -Derechos Humanos de las mujeres en México; -Detención de niños en México; -Desapariciones Forzadas y Ejecuciones Sumarias en México; -Explotación infantil en México; -Implicación del narco en el feminicidio de Ciudad Juárez; -Feminicidio; -Feminicidio en Guatemala. Similitudes con México; -Libertad de expresión en México; -Posicionamiento del Parlamento Europeo contra el feminicidio en América Latina; -Prácticas tradicionales que afectan a la salud de mujeres y niñas en México; -Situación de los defensores de los Derechos Humanos; -Tortura y detención arbitraria en México; -Tráfico de niños, prostitución y pornografía infantil en México; -Violaciones de los derechos humanos de los/as obreros/as migrantes en México y -Violencia contra las mujeres.

-Documentos e informes de México y Estados Unidos sobre la historia reciente de México: -La Guerra Sucia en México; La problemática de la Seguridad en la frontera Sur de México; Los abusos militares en Chiapas y la problemática del ejército mexicano.

Asimismo se contrastó con una amplia bibliografía clasificada temáticamente por los siguientes apartados temáticos: -Combate al narcotráfico; -Corrupción; -Crimen Organizado; -Derechos Humanos; -Ejército; -Feminicidio; -Historia de México;-Impunidad; Maquila; -Narcotráfico; -Pederastia; -Sectas Narcosatánicas y Santa Muerte;Seguridad; -Situación social de las mujeres, -Sociedad mexicana y-Tortura.

2. Análisis de contenido. Se han estudiado un total de 9.000 noticias de los tres medios de comunicación mencionados (Proceso, Contranlína y CIMAC) seleccionadas por el tratamiento del narcotráfico o del feminicidio de Ciudad Juárez. Se realizó el análisis de contenido en dos fases:

Fase A. Estudio de la aplicación de los valores noticia tradicionales teniendo como base las concepciones teóricas anteriormente expuestas sobre los valores noticia y la realidad de los medios y el contexto mexicano. Se han revisado los valores noticia a través del empleo de la ficha de análisis que figura en el anexo sobre las noticias de tres medios analizados (incluida en el anexo I) pretende destacar los aspectos principalmente afectados por esta reconfiguración, y contraponer a la vez el énfasis que unos medios ponen en unos factores sobre otros. Como indicábamos anteriormente se establecieron una serie de categorías a partir de la revisión teórica, del contexto y del análisis de los medios analizados que se resumen a continuación:

${ }^{3}$ Debido al gran número de ellas se hace imposible mencionarlas aquí, si bien pueden ser consultadas en la siguiente tesis doctoral: Gallur Santorum, Santiago. Hijas del Silencio. El contexto de los asesinatos y desapariciones de mujeres en Ciudad Juárez. Santiago de Compostela, Universidad de Santiago de Compostela, (12-Diciembre) 2011. 
1) Actualidad: Todo lo que esté cerca del presente es actualidad, tanto el pasado como el futuro próximo. La máxima del periodismo es la simultaneidad, es decir, que coincida el momento en el que ocurre la noticia con la emisión en directo de la misma.

2) Novedad: Conocimiento que el público tiene del acontecimiento. Se suele confundir con actualidad porque lo más actual es novedad para el público.

3) Importancia: La importancia es relativa. Un hecho puede ser importante por los siguientes factores:

1) Cuanto mayor impacto tenga sobre la zona de difusión.

2) Según el número de personas implicadas.

3) Si el acontecimiento es significativo.

4) Según evolucione el acontecimiento.

5) En función del grado o nivel jerárquico de los implicados.

6) Por la notoriedad del sujeto.

7) Según el interés de la audiencia: se seleccionan acontecimientos que interesen a la audiencia del medio. A la vez, podemos decir que los intereses mayoritarios son:

1) Proximidad geográfica.

2) Proximidad social.

3) Lo extraordinario.

4) Preferencia temática.

5) Interés humano.

Así, estos criterios, que son comúnmente aceptados por los medios de comunicación, suelen funcionar eficazmente en un número importante de ocasiones de un modo simultáneo. Sin embargo, estos criterios - unidos a los factores de publicación- pueden mostrar intencionadamente una pequeña parte de la realidad, aquella que conviene y aceptan tanto los medios como el conjunto de la sociedad de la zona concreta en la que se sitúe dicho medio. Es decir, que mientras se muestra esa pequeña porción del mundo, otra gran porción de la realidad queda silenciada y no es conocida para la sociedad, poniendo en entre dicho el derecho fundamental a la información.

Esta circunstancias, en situaciones extremas puede dar lugar a un silenciamiento de acontecimientos realmente importantes y que al status quo del país en concreto no lo conviene difundir. Es precisamente algo muy similar a lo anterior lo que está ocurriendo en la actualidad con la situación de violencia extrema que está sucediendo en México desde hace ya algunos años debido en parte a la conocida como "guerra contra el narco". Así, los cárteles de la droga que han atemorizado al país entero a base de exponer cabezas cortadas y cuerpos decapitados en la vía pública, a la vez han impuesto en los medios de comunicación un silencio obligado, fundamentado en el terrible lema "plata o plomo": o te dejas corromper y silencias las noticias que te indique, o haces tu trabajo periodístico muriendo asesinado como consecuencia directa. Esta macrabra pero eficaz estrategia comunicativa del narco provoca que la mayoría de medios de comunicación difundan únicamente aquellas noticias que no están vetadas por el narco y sus socios. Por lo tanto, el resultado evidente es un panorama de des- 
información generalizado debido a que muchos medios se dedican exclusivamente a contar muertos, mientras sacan en portada las cabezas cortadas o los cuerpos decapitados arrojados por el propio narco en muchas zonas del país. Así, la situación de terror colectivo se difunde y perpetúa, a la vez que una gran cantidad de noticias importantes son silenciadas.

Por todo esto los valores noticias, en situaciones excepcionales como el caso mexicano actual, no son suficientes para garantizar el derecho a la información de la ciudadanía, y por lo tanto se convierten más bien en medios perfectos que justifican el silenciamiento de hechos importantes. Así, se hace necesario la utilización de una serie de factores complementarios de noticiabilidad, que pueden funcionar de apoyo a los valores noticia, y que permitirían sacar a la luz hechos relevantes en contextos en los que la libertad informativa está cuestionada por situaciones de violencia extrema y amenazas hacia los periodistas y que indicamos en el siguiente apartado.

Fase B. Propuesta de nuevos factores de noticiabilidad y comprobación de su validez en la información publicada. Una vez realizado este estudio se identificaron una serie de valores noticia que hipotéticamente recogerían los que son utilizados en esta situación de conflicto y se comprobó su validez en las mismas informaciones estudiadas en la primera parte de este análisis de contenido. Si bien estos factores no estarían presentes en su totalidad en los tres medios, sí lo están una combinación concreta de los mismos que es la que a su vez acaba diferenciando su producción informativa $\mathrm{y}$ hace que sus noticias sean distintas en cada uno de los tres. Estas actitudes o factores complementarios de noticiabilidad serían los siguientes:

$1^{\circ}$ ) Invertir los criterios sociales que marcan la noticiabilidad, es decir, cambiar las tendencias sociales que señalan lo que es considerado noticia, utilizando valores que no necesariamente son respaldados por la mayoría de la población.

$\left.2^{\circ}\right)$ Dar "voz" a los protagonistas de las situaciones, independientemente del cargo o posición social de la persona.

$3^{\circ}$ ) Priorizar la utilización como fuentes de las víctimas de las situaciones, independientemente de su posición socioeconómica.

$\left.4^{\circ}\right)$ Los sujetos con algo que decir sobre el tema a tratar deben ser considerados como fuentes, no discriminando por ideología, nivel socioeconómico o posicionamiento político.

$5^{\circ}$ ) Serán considerados como fuentes hombres o mujeres.

$\left.6^{\circ}\right)$ Priorizar la voz de las mujeres, fomentando a su vez la visibilización de su versión de los hechos en entornos de comunicación, a través de la difusión de noticias protagonizadas o contadas por ellas.

Las piezas informativas analizadas fueron las siguientes:

-Todas las noticias sobre el narcotráfico en México publicadas por Proceso desde su creación en 1976 hasta el 2010 (fecha en la que se marcó el límite de la investigación). Es necesario señalar que no es hasta 1989-1990 cuando la revista de investigación comienza a llevar a cabo una cobertura periódica de esta temática, llegando a tener en la actualidad una sección propia.

-Todas las noticias sobre el narcotráfico en México publicadas por Contralínea desde 
su creación en el 2001 hasta el 2010 (fecha en la que se marcó el límite de la investigación), ya que esta revista de investigación ha dedicado desde su nacimiento una gran parte de sus recursos a la investigación del narcotráfico y la corrupción asociada.

-Todas las noticias sobre el feminicidio de Ciudad Juárez publicadas por la agencia de noticias CIMAC (Comunicación e Información de la Mujer AC) desde 1999 hasta el 2010 (fecha en la que se marcó el límite de la investigación). Hay que destacar que este es el único medio de comunicación que ha llevado un seguimiento periódico de todos los hechos que rodean al feminicidio de Ciudad Juárez, así como su contextualización histórica.

3. Entrevistas. Para poder llevar a cabo el proceso de contraste y verificación de la calidad de las informaciones publicadas por Proceso, Contralínea y CIMAC, como parte fundamental de esta investigación, han constituido un papel esencial para el desarrollo de la misma las entrevistas realizadas a distintos expertos en temas vinculados de una u otra forma con el narcotráfico y el feminicidio de Juárez. La relación de las entrevistas así como el guión básico común de las mismas se presenta en el anexo del artículo. Con la investigación entenderemos los efectos y posibilidades periodísticas de esta nueva forma de percibir la labor de los medios de comunicación que deriva de un claro compromiso social y cuestionamiento de las versiones y las fuentes oficiales socialmente aceptadas.

Por último, las entrevistas realizadas a los responsables de la información de los medios analizados y a grandes expertos en el narcotráfico mexicano y en el feminicidio de Ciudad Juárez (se especifican en el anexo II), nos permitirán (además de contrastar la calidad de las informaciones proporcionadas por dichos medios) poder comprender la importancia que esta inversión de los valores noticia tiene en la producción informativa, y por tanto en la calidad de la información que recibe la sociedad.

\section{Análisis de datos}

Tras el análisis detenido de las informaciones publicadas en los medios estudiados podemos concretar la dislocalición de los valores noticia tradicionales y su adaptación a la situación de conflicto analizada. De este modo, los tres medios convierten en noticia hechos que no figuran en la mayoría de los medios de comunicación y, sobre todo, permiten a Proceso, Contralínea y CIMAC, romper claramente la barrera de la noticiabilidad tradicional y trabajar con los acontecimientos desde una perspectiva estructural y no solamente coyunturalmente. Los seis factores inicialmente propuestos funcionan como una estructura definida y pensada para cambiar el panorama informativo de los medios pudiendo incluir con normalidad temáticas tan complejas y difíciles de cubrir para el periodista como el narcotráfico o el feminicidio de Ciudad Juárez (en la grave situación de conflicto que vive México), sin que determinados factores de publicación o prejuicios sociales (como el machismo o el racismo en el caso de sucesos que afectan a determinadas comunidades indígenas, etc.) funcionen como barreras. A continuación se resumen los principales resultados de análisis de datos llevado a cabo en cada uno de los casos. 
Entre todas las noticias publicadas por Proceso identificamos el uso de dos factores de noticiabilidad de los seis expuestos al principio de este artículo, que son los siguientes:

$1^{\circ}$ ) Se invierten los criterios sociales que marcan la noticiabilidad, es decir, que se cambian las tendencias sociales que señalan lo que es considerado noticia.

$2^{\circ}$ ) Tienen "voz" los protagonistas de las situaciones, independientemente del cargo o posición social de la persona.

De este modo Proceso consigue por una parte, sacar a la luz hechos vinculados con el narcotráfico y la corrupción de las autoridades, a la vez que sitúa como fuentes a protagonistas de los hechos que normalmente en los medios son invisibilizados al no ser considerados como origen válido de la información. Esto se debe principalmente a que la fuente suele ser valorada por su cargo o posición socioeconómica. Así, en casos concretos en los que los protagonistas de los hechos no solo son pobres sino que son marginados socialmente por su género, etnia y tendencia ideológica, estos testimonios nunca salen a la luz, provocando así un sesgo en la realidad comunicada a través de los medios. Por tanto, a pesar de que Proceso solo suele utilizar dos de los seis factores de noticiabilidad mencionados, los resultados son realmente notables, debido a que los lleva implementando de forma sistemática en la cobertura de las noticias sobre el narcotráfico mexicano que con los valores noticia aplicados para situaciones no conflictivas nunca entrarían a formar parte de la agenda mediática.

Gracias a todo lo mencionado la revista Proceso ha conseguido sacar a la luz en estos más de 30 años de existencia, un sin fin de tramas de corrupción vinculadas al narcotráfico, que han destapado la enorme penetración del narco en el estado mexicano y la protección que las propias autoridades le ha brindado en infinidad de ocasiones, así como la gran violencia derivada del narcotráfico. Por ello se hace necesario destacar que la inversión de los valores noticia llevada a cabo por Proceso, además de permitir que una grandísima cantidad de hechos tremendamente relevantes se hayan convertido en noticia, ha creado una tendencia en el periodismo de investigación mexicano que ha sido llevada a su máxima expresión por medios como Contralínea o CIMAC.

En el análisis llevado a cabo de las noticias publicadas por Contralínea identificamos principalmente el uso de cuatro de los valores de los seis expuestos al principio de este artículo, que son los siguientes:

$\left.1^{\circ}\right)$ Se invierten los criterios sociales que marcan la noticiabilidad, es decir, que se cambian las tendencias sociales que señalan lo que es considerado noticia.

$2^{\circ}$ ) Tienen "voz" los protagonistas de las situaciones, independientemente del cargo o posición social de la persona.

$3^{\circ}$ ) Se prioriza la utilización como fuente de las víctimas de las situaciones, independientemente de su posición socioeconómica.

$4^{\circ}$ ) Todos los sujetos con algo que decir sobre el tema a tratar se contemplan como fuentes, no discriminando por ideología, nivel socioeconómico o posicionamiento político. 
El uso de estos cuatro factores tiene como resultado una visibilización de hechos relacionados con el narcotráfico y su violencia asociada, en los que se utiliza como fuente a los propios protagonistas o víctimas de los acontecimientos. Si bien, esto se daba también en Proceso, Contralínea da un paso más, debido a lo extremo de la temática que trata y a la gran importancia de los datos que saca a la luz. Así, utilizando los cuatro criterios de noticiabilidad que consiguen la inversión de los valores noticia, el medio saca a la luz hechos tan importantes como que las propias autoridades mexicanas están llevando a cabo campañas de control social a través del uso de la violencia extrema en zonas con fuerte presencia de grupos como el APPO. Sin embargo, lo más importante es que el medio ha conseguido documentar que algunas autoridades están utilizando a grupos de sicarios de los cárteles apoyados por el propio ejército mexicano para enfrentarse a la guerrilla. En concreto el medio utiliza como fuente a los propios guerrilleros e incluso a civiles testigos de dichos enfrentamientos y del vínculo entre autoridades y cárteles en la lucha contra la guerrilla.

Este tipo de noticias, que sería imposible publicar en otro medio de comunicación mexicano, en Contralínea se han convertido en su sello de identidad, marcadas por una profunda crítica hacia la corrupción y protección del narco por parte de las propias autoridades. Es necesario destacar por tanto, que normalmente los medios de comunicación no suelen tomar como fuentes a miembros de grupos guerrilleros, debido a un claro posicionamiento ideológico dentro de la línea editorial en contra de la insurrección armada llevada a cabo en ciertas zonas de México y América Latina. Sin embargo, el hecho de utilizar a miembros guerrilleros y testigos de los enfrentamientos como fuente, no implica el apoyo ideológico a la guerrilla, sino más bien la búsqueda de completar un espectro de la realidad que normalmente nunca es cubierto debido a que se rechaza como fuente a una parte de los protagonistas de los hechos, a la vez que se difunde las contradictorias versiones de las voces oficiales: las propias autoridades.

La utilización de los protagonistas de los hechos como fuentes, a pesar de que es algo básico en el periodismo teórico, en la práctica se convierte en realidad en algo poco común en los medios. Si bien se justifica basándose en la línea editorial del medio en cuestión, esta tendencia oculta un ejercicio de "mala paxis" periodística donde los acontecimientos se exponen a través de las voces oficiales y de las fuentes que se encargan de velar por la estabilidad del statu quo. Si todo esto lo aplicamos a la cobertura de los sucesos relacionados con el narcotráfico, teniendo en cuenta lo tremendamente peligroso que supone el periodismo de este tipo que engloba autocensura y riesgo real para la vida del periodista, se acaba produciendo un grandísimo sesgo de la realidad. Sin embargo, Contralínea rompe esta tendencia ofreciendo noticias de una altísima calidad, caracterizadas por el uso como fuentes de los protagonistas de los hechos, independientemente de cuál sea su adscripción política o clase social. El resultado es un periodismo de investigación tremendamente crítico con la versión de las autoridades y que no hace campaña política de una idea sino que pretende ofrecer un panorama más amplio de la realidad (del narcotráfico): aquellos hechos que normalmente son negados y ocultados por las propias autoridades.

En el análisis de las noticias públicadas por $C I M A C$ en la cobertura que ha llevado a cabo sobre el feminicidio de Ciudad Juárez, hemos podido identificar el uso de los 
seis factores de noticiabilidad expuestos al principio de este artículo, que son los siguientes:

$\left.1^{\circ}\right)$ Se invierten los criterios sociales que marcan la noticiabilidad, es decir, que se cambian las tendencias sociales que señalan lo que es considerado noticia.

$2^{\circ}$ ) Tienen "voz" los protagonistas de las situaciones, independientemente del cargo o posición social de la persona.

$3^{\circ}$ ) Se prioriza la utilización como fuente de las víctimas de las situaciones, independientemente de su posición socioeconómica.

$4^{\circ}$ ) Todos los sujetos con algo que decir sobre el tema a tratar (narcotráfico o feminicidio) se contemplan como fuentes, no discriminando por ideología, nivel socioeconómico o posicionamiento político.

$5^{\circ}$ ) Son considerados como fuentes hombres o mujeres por igual.

$6^{\circ}$ ) Se prioriza la voz de las mujeres, fomentando a su vez la visibilización de su versión de los hechos en entornos de comunicación a través de la difusión de noticias protagonizadas o contadas por ellas.

CIMAC practica un tipo de periodismo bien distinto al estilo de Proceso, y con algunas similitudes al de Contralínea, pero que tiene como característica fundamental el género. Así, mientras Proceso y Contralínea llevan a cabo una inversión de los valores noticia en la información relacionada con el narcotráfico, CIMAC lo hace en la cobertura del feminicidio de Ciudad Juárez. A pesar de las aparentes diferencias que podrían entenderse como derivadas de este tipo de temática, en realidad el medio hace una cobertura informativa que tiene en cuenta cualquier problemática que afecta a las mujeres. Así, en el caso del feminicidio, CIMAC ha conseguido sacar a la luz a través de algunas sus noticias el vínculo existente entre el narco y los asesinatos y desapariciones de mujeres ocurridos en Ciudad Juárez. Debido a esto, en casos concretos el medio hace a la vez una cobertura informativa conjunta del feminicidio y del narcotráfico, que se puede entender como la información de la implicación que personas vinculadas al narco han tenido en la comisión y protección de dichos crímenes.

Como vemos en el esquema de factores de noticiabilidad utilizados, CIMAC ha usado aquellos factores utilizados también por Proceso (dos) y Contralínea (cuatro), y ha añadido dos más que se centran en la visión de género. Así, mediante la equiparación de hombres y mujeres como fuentes y de la priorización de la voz de las mujeres como protagonistas de los hechos, CIMAC ha perfeccionado la inversión de los valores noticia, logrando sacar a la luz los acontecimientos de los que ningún otro medio informa.

Para entender la gran importancia que tiene para el periodismo la cobertura del feminicidio llevada a cabo por esta agencia de noticias, debemos analizar el espectro habitual de la realidad cubierta por una gran mayoría de los medios. Así, a pesar de que el mundo está formado por hombres y mujeres, y ambos suelen ser protagonistas de los hechos a partes iguales, habitualmente la voz de las mujeres está mucho menos considerada como fuente que la de los hombres. Esta situación se agrava sobremanera si las mujeres protagonistas son de estatus socioeconómico bajo y más aún si tienen rasgos étnicos en países con sociedades claramente racistas. Por todo ello, la versión 
de la realidad en la que las mujeres son protagonistas de los hechos, simplemente está fuera del espectro informativo ofrecido por la mayoría de los medios de comunicación. Por lo tanto, estamos siendo informados frecuentemente de una realidad en la que la mitad de la sociedad, las mujeres, son frecuentemente excluidas como fuentes incluso cuando son víctimas o protagonistas de los hechos publicados.

CIMAC ha roto definitivamente con esta exclusión de las mujeres de la agenda mediática, haciendo justo lo contrario a lo hecho tradicionalmente: priorizando la voz de las mujeres como fuentes. Esto ha provocado la salida a la luz de un si fin de temáticas que hasta el momento no eran tenidas en cuenta por los medios. Así, la inversión de los valores noticia, apoyada en los factores de noticiabilidad mencionados previamente, permite que se conozcan a través de algunas de las noticias publicadas en CIMAC hechos tan graves como la posible implicación del narcotráfico en una parte de los crímenes del feminicidio de Ciudad Juárez. Esto ha sido debido, fundamentalmente, a la priorización como fuente de las voz de las familias de las víctimas y de aquellos expertos y personalidades que han aportado pruebas irrefutables que señalan una gran red de corrupción y poder detrás de una parte de dichos crímenes.

Por todo lo anterior podemos considerar que CIMAC ha conseguido aplicar hasta sus últimas consecuencias la inversión de los valores noticia, dentro de una perspectiva de género, que no pretende excluir a los hombres de la realidad, sino incluir en ella a las mujeres. De esta forma, las mujeres se convierten a voces autorizadas al ser víctimas o protagonistas de acontecimientos que de otra manera estarían silenciados. La cobertura del feminicidio de Ciudad Juárez llevada a cabo por esta agencia de noticia es por tanto el mejor ejemplo de inversión de valores noticia, y de la infinidad de posibilidades informativas que ofrece este modo de entender el periodismo.

\section{Conclusiones}

La atención que el medio de comunicación presta a los conflictos estructurales proporciona uno de los mejores criterios para evaluar el rigor y la profundidad del discurso público. No hay que darla por descontada: operan contra ella la necesidad de informar y comentar de inmediato hechos noticiables que han de ser rápidamente interpretados y la presunción de que la audiencia tiene mucho más interés por los relatos fuertemente personalizados que por el análisis estructural.

Muchas veces los conflictos estructurales son explicados periodísticamente como si fueran meros conflictos conyunturales; muy pocas veces el periódico se ocupa de aquellos conflictos estructurales que por su propia configuración no aseguran el "interés" periodístico (en tanto que no generan hechos noticiables espectaculares) (BoRRAT, 1989: 24). Por lo tanto, hay un sesgo de la realidad al ofrecernos únicamente una muy pequeña parte de la misma.

Este "error" derivado de la imposibilidad de informar de todo lo que sucede, ha sido aceptado y justificado en infinidad de ocasiones apoyándose en la línea editorial de los medios y en mecanismos de selección de noticias, como son los valores noticias. El problema viene cuando la propia sociedad adquiere la consciencia (debido a situaciones de grave conflicto como es la que vive en la actualidad en México), de que normalmente muchos medios no ofrecen una serie de acontecimientos por la imposi- 
bilidad de incluirlos todos, sino que informan de unos hechos que incluyen en la agenda informativa para evitar así que se conozcan otros.

Esta investigación ha permitido comprobar que medios tan distintos como Proceso, Contralínea y CIMAC han llevado a cabo una dislocación concreta de los valores noticia tradicionales, apoyándose en una serie de factores, con el principal objetivo de informar de aquellos hechos que son silenciados por otros muchos medios de comunicación. Estos factores están presentes en la actualidad en tres medios mexicanos, Proceso, Contralínea y CIMAC, que han destacado por la cobertura del narcotráfico (los dos primeros) y del feminicidio de Ciudad Juárez (el tercero), sacando a la luz así noticias sobre estos temas que no son difundidas por casi ningún otro medio. Por lo tanto, estos criterios mencionados previamente son esenciales para dar a conocer hechos, invisibilizados por la mayoría de los medios de comunicación, pero que tienen mucha importancia para la sociedad civil ${ }^{4}$.

Las consecuencias para el ejercicio del periodismo son directas, muestran que otro tipo de periodismo es posible, incluso en situaciones en la que el oficio de informar puede suponer la muerte, como es el escenario de "guerra contra el narco" que se lleva viviendo en México desde 2006. Así, enfrentándose al terrible lema "plata o plomo" del narco que ofrece al periodista la brutal decisión de tener que elegir entre morir (el plomo hace referencia a la bala) o vivir en la riqueza (la plata hace referencia a las grandes ganancias que aporta la corrupción del narco), los profesionales de la información de medios como Proceso, Contralínea o CIMAC se posicionan de manera contundente y eligen una tercera opción: el hacer bien su trabajo para informar a la sociedad, aunque esto les cueste la vida.

\section{Referencias bibliográficas}

ARMENTIA, José Ignacio; CAMINOS, José María (2003): Fundamentos de periodismo impreso. Barcelona, Ariel.

BORRAT, Héctor (1989): El periódico, actor político. Barcelona, Gustavo Gili.

CASALS CARRO, María Jesús (2005): Periodismo y sentido de la realidad. Teoría y análisis de la narrativa periodística. Madrid, Fragua.

DIEZHANDINO NIETO, María Pilar (1994): El quehacer informativo: el arte de escribir un texto periodístico. Bilbao, Servicio Editorial de la Universidad del País Vasco.

DIJK, Teun A. van (1990): La noticia como discurso. Comprensión, estructura y producción de la información. Barcelona, Paidós.

EILDERS, Christiane (2006): "News factors and news decisions. Theoretical and methodological advances in Germany". Communications: The European Journal of Communication Research, volume 31, number 1, pp. 5-24.

FONTCUBERTA, Mar de (1993): La noticia. Pistas para percibir el mundo. Barcelona, Paidós.

${ }^{4}$ Se incluyen en el anexo III algunos ejemplos de noticias de estos casos. 
GALGUNG, Johan; RUGE, Mari (1965): "The structure of foreign news: the presentation of the Congo, Cuba and Cyprus crises in four Norwegian newspapers". Journal of International Peace Research, n. 2, pp. 64-91.

GANS, Herbert J. (1980): Deciding what's news. A study of CBS Evening News. NBC nightly New, Newsweek and Time. Nueva York, Pantheon Books.

KEPPLINGER, Hans Mathias; EHMIG, Simone Christine (2006): "Predicting news decisions. An empirical test of the two-component theory of news selection". Communications: The European Journal of Communication Research, volume 31, number 1, pp. 25-40.

LÓPEZ GARCÍA, Xosé; TÚÑEZ LÓPEZ, Miguel (1995): Redacción en prensa: a noticia. Santiago de Compostela, Edicións Lea.

MUÑOZ-TORRES, Juan Ramón (2002): Por qué interesan las noticias. Un estudio de los fundamentos del interés informativo. Barcelona, Herder.

RUHRMANN, G.; WOELKE, J.; MAIER, M; DIEHLMANN, N (2003): El valor de noticias en la televisión alemana: un modelo para validar los factores noticiosos, Opladen, Leske/Budrich.

SCHWARZ, Andreas (2010): "La teoría de los valores noticiosos y su validez externa: cómo influyen los factores noticiosos en la cobertura de las noticias internacionales en la prensa mexicana", en HERNÁNDEZ RAMÍREZ, Elena (coord.): Estudios sobre periodismo. Marcos de interpretación para el contexto mexicano, Guadalajara (Mexico), Editorial de la Universidad de Guadalajara, p. 19-55.

SERRANO, José Francisco (coord.) (2003): Redacción para periodistas: informar e interpretar. Barcelona, Ariel.

STAAB, J.F. (1990): "The role of news factors in news selection: a theoretical reconsideration". European Journal of communication, no 5, p. 423-443.

WARREN, C. (1979): Generos periodísticos informativos. Barcelona, ATE.

\section{Anexos}

\subsection{Anexo I. Entrevistas realizadas}

Las entrevistas llevadas a cabo para la investigación fueron las siguientes:

- María Josefina Morales Fernández, Doctora en Estudios Latinoamericanos por la UNAM (Universidad Nacional Autónoma de México), autora de la tesis doctoral "Maquila 1980-2006. México, Centroamérica y La República Dominicana" y profesora de la UNAM. Mayo de 2010 en Santiago de Compostela,

- José Luis Soberanes, Ex Presidente da la Comisión Nacional de Derechos Humanos (CNDH) de México y Catedrático de Derecho de la UNAM. 16 de agosto de 2010, en México DF.

- Nancy Flores y Zósimo Camacho, editora y jefe de información respectivamente de la revista de periodismo de investigación Contralínea. 17 de agosto de 2010 en México DF. 
- Tomás Santiago Serrano Pérez, Director General del Programa de Presuntos Desaparecidos de la Comisión Nacional de Derechos Humanos (CNDH) de México, encargado de realizar las investigaciones sobre las desapariciones de la Guerra Sucia y de llevar todas las investigaciones sobre los asesinatos y desapariciones de mujeres en Ciudad Juárez. 18 de agosto de 2010 en México DF.

- Lucía Lagunas, Directora de la agencia de noticias Comunicación e Información de la Mujer A.C., (CIMAC). 20 de agosto de 2010 en México DF.

- Encuentro con un Policía Judicial de México DF. 21 de agosto de 2010.

- Rosario Novoa, Profesora de Postgrado que trabaja en la Secretaría de Seguridad Pública en México DF y que ha trabajado en Ciudad Juárez y escrito sobre el feminicidio. 24 de agosto de 2010 en México DF.

\subsection{Anexo II. Informaciones relevantes}

Ofrecemos a continuación una serie de informaciones significativas publicadas por cada medio:

\section{Proceso:}

Balboa, Juan y Manuel Robles. "La huella de su mano dura" [en línea]. Proceso, 20 de octubre de 1990.

Cobián, Felipe y Raúl Monge. "Soldados en matanzas de Guadalajara y Chihuahua" [en línea]. Proceso, 26 de junio de 1993.

Correa, Guillermo e Ignacio Ramírez. "Colección de gobernadores ineptos, abusivos y represivos" [en línea].

Correa, Guillermo y Alejandro Gutiérrez. "'Quemaron las casas, saquearon, nos torturaron"”' [en línea]. Proceso, 28 de noviembre de 1992.

Díaz, Gloria Leticia. "Tijuana: los soldados torturadores" [en línea]. Proceso, 15 de febrero de 2010.

Dávila, Patricia. "Juárez: Entre sicarios y soldados" [en línea]. Proceso, 29 de agosto de 2009.

Esquivel, J. Jesús. "Los Zetas" y los "Gatekeepers", aliados fronterizos" [en línea]. Proceso, 23 de febrero de 2008.

Gutiérrez, Alejandro. "Enfrentamientos con militares en la sierra de chihuahua; familias enteras abandonan la zona" [en línea]. Proceso, 30 de abril de 1994.

Gutiérrez, Alejandro y Guillermo Correa. "El jefe del cuerpo militar acusado de represión, la niega y culpa a los indígenas de narcos" [en línea]. Proceso, 28 de noviembre de 1992.

Mergier, Anne Marie. "Desproporción del contrataque militar" [en línea]. Proceso, 8 de enero de 1994.

Monge, Raúl. "Justicia militar precipitada... con antiguos expedientes" [en línea]. Proceso, 2 de septiembre de 2000.

Monge, Raúl. "Tortura, delitos fabricados, crímenes políticos e impunidad, rutinas del Estado de Guerrero" [en línea]. Proceso, 10 de julio de 1995. 
Ramírez, Ignacio. "En Guerrero cunde el desprecio de las Fuerzas Públicas a los derechos humanos" [en línea]. Proceso, 3 de noviembre de 1990.

Ravelo, Ricardo. "El caso DC-9: PFP y Conagua, Enredados" [en línea]. Proceso, 30 de julio de 2006.

Rodríguez García, Arturo. "La Hermandad de Comandantes" [en línea]. Proceso, 17 de mayo de 2008.

Ortiz Pinchetti, Francisco. "Secuestros, asaltos, homicidios, linchamientos y descrédito de los cuerpos de seguridad" [en línea]. Proceso, 6 de abril de 1996.

Valdez Reycen, Jorge. "Admite el secretario de la defensa que hubo excesos en Chihuahua; promete investigar" [en línea]. Proceso, 28 de noviembre de 1992.

Villamil, Jenaro. "Solicita Senado informe sobre "escuadrones de la muerte"" [en línea]. Proceso, 23 de septiembre de 2010.

\section{Contralínea:}

Ana Lilia. "Encubre PGR al cártel de Pénjamo" [en línea]. Contralínea, $1^{\mathrm{a}}$ quincena de octubre de 2006.

Camacho, Zósimo. "Montaña de Guerrero pobreza y militarización" [en línea]. Contralínea, $1^{\text {a }}$ quincena de enero de 2007.

Esquivel, J. Jesús. "Washington aplaude, pero...” [en línea]. Proceso, 6 de enero de 2007.

Flores, Nancy. "Contrainsurgencia, objetivo militar" [en línea]. Contralínea, $1^{\mathrm{a}}$ quincena de febrero de 2007.

Flores, Nancy. "Plan México 2030 "acotar" crimen organizado, no exterminarlo" [en línea]. Contralínea, 28 de febrero de 2010

Guzmán, Armando. "Pérez, Ana Lilia. "Zetas y Kaibiles contra la APPO “ [en línea]. Contralinea, $2^{\mathrm{a}}$ quincena de octubre de 2006.

Laguna, Mauricio. "Crimen organizado produce el 40\% del PIB“" [en línea]. Contralínea, $2^{\mathrm{a}}$ quincena de julio de 2007.

Lilia. "Maras, los soldados del narcotráfico" [en línea]. Contralínea, $1^{a}$ quincena de marzo de 2006.

Martínez, Alba. "Ulises y paramilitares aterrorizan a la mixteca oxaqueña” [en línea]. Contralínea, $1^{\text {a }}$ quincena de junio de 2008.

Pérez, Ana Lilia. "Alianza de cárteles de México y Guatemala” [en línea]. Contralínea, $1^{\text {a }}$ quincena de marzo de 2006.

Pérez, Ana Lilia. "Kaibiles, negocio desde el infierno" [en línea]. Contralínea, $2^{\mathrm{a}}$ quincena de enero de 2007.

Pérez, Ana Lilia. "Los Kaibiles mexicanos" [en línea]. Contralínea, 2a quincena de enero de 2007.

Pérez, Ana Lilia. "Manual de terrorismo" [en línea]. Contralínea, n 14, julio de 2003. Pérez, Ana Lilia. "Maras, los soldados del narcotráfico" [en línea]. Contralínea, $1^{\text {a }}$ quincena de marzo de 2006. 
Pérez, Ana Lilia. "Militares y paramilitares en Chiapas" [en línea]. Contralínea, $2^{\mathrm{a}}$ quincena de mayo de 2006.

Pérez, Ana Lilia. "Militares y paramilitares en Chiapas" [en línea]. Contralínea, $2^{\mathrm{a}}$ quincena de mayo de 2006.

Pérez, Ana Lilia. "Zetas y Kaibiles contra la APPO “ [en línea]. Contralínea, 2 quincena de octubre de 2006.

Ramírez, Erika. "Guerra sucia contra la APPO" [en línea]. Contralínea, $1^{\text {a }}$ quincena de diciembre de 2006.

Rees, Ernesto. "El fantasma de la guerrilla" [en línea]. Contralínea, $2^{\text {a }}$ de octubre de 2006.

Reyez, José . "Revelaciones del joyero del Señor de los Cielos" [en línea]. Contralínea, 25 de abril de 2010.

Torres, Jorge. "El imperio de la droga" [en línea]. Contralínea, no 16, septiembre de 2003.

Vilchis, José. "La impunidad del fuero militar" [en línea]. Contralínea, $\mathrm{n}^{\circ} 7$, julio de 2002.

\section{CIMAC:}

"Deseable, que Fiscalía acabe definitivamente con feminicidio" [en línea]. CIMAC, 30 de julio de 2007.

González, María De la Luz. "Crea PGR, Fondo para familias de víctimas de feminicidio" [en línea]. CIMAC, 31 de mayo de 2005.

González, Román. "Marcan a Ciudad Juárez pobreza, narcotráfico y violencia" [en línea]. CIMAC, 28 de marzo de 2003.

González, Román. "Pasividad de autoridades y omisión de partidos por Feminicidio" [en línea]. CIMAC, 11 de septiembre de 2004.

Gutiérrez, Alma Angelina. "Intolerables los asesinatos de mujeres en Ciudad Juárez: Beatriz Paredes" [en línea]. CIMAC, 18 de marzo de 2002.

Jarquín Edgar, Soledad. "El gobierno mexicano imposibilitado para esclarecer el feminicidio: Epikeia" [en línea]. CIMAC, 25 de abril de 2003.

"Llevará Italia el Caso Juárez a la cumbre UE-América Latina" [en línea]. CIMAC, 18 de diciembre de 2003.

Magally, Silvia. "Conoció Heyzer el terror que se vive en Ciudad Juárez" [en línea]. CIMAC, 3 de diciembre de 2002 [en línea]. CIMAC, 3 de diciembre de 2002.

Magally, Silvia. "En Ciudad Juárez todo puede suceder: hasta la impunidad de 268 crímenes" [en línea]. CIMAC, 20 de mayo de 2002.

Maya, Rafael. "Narcotráfico y degradación social causa de feminicidios de Juárez" [en línea]. CIMAC, 12 de septiembre de 2002.

Magally, Silvia. "Pide Marta Lamas intervención policiaca internacional en el caso Juárez" [en línea]. CIMAC, 7 de marzo de 2002.

Maya, Rafael. "Ganadora de Ariel culpa a gobierno por feminicidio en Juárez" [en línea]. CIMAC, 9 de abril de 2002. 
Maya, Rafael. "Intenta Fox desprestigiar al gobierno de Chihuahua: Sauri” [en línea]. CIMAC, 29 de noviembre de 2002.

Maya, Rafael. "Lamentan politización de los asesinatos de mujeres en Juárez" [en línea]. CIMAC, 28 de noviembre de 2002.

Maya, Rafael. "Marco jurídico vigente oculta discriminación contra mujeres" [en línea]. CIMAC, 10 de agosto de 2004.

Maya, Rafael. "Pide comisionada deslinde de autoridades con delincuencia en Juárez" [en línea]. CIMAC, 26 de julio de 2004.

Martínez, Martha. "Sin sustento tráfico de órganos en Ciudad Juárez" [en línea]. CIMAC, 9 de mayo de 2003.

"Necesario, atender rezago social para evitar crímenes en Juárez: Esther Chávez" [en línea]. CIMAC, 25 de noviembre de 2001.

Pérez, Ana Lilia. "Kaibiles, escuela de asesinos" [en línea]. Contralínea, $2^{\mathrm{a}}$ quincena de enero de 2007.

Ruiz, Miriam. "Gobierno, cómplice de feminicidio en Juárez: juristas de UNAM" [en línea]. CIMAC, 22 de enero de 2002.

"Seguirá feminicidio mientras persista cultura misógina" [en línea]. CIMAC, 30 de julio de 2004.

\subsection{Anexo III. Extractos de noticias con la transformación de los valores noticia} A continuación se muestran unos extractos de noticias difundidas por dos de estos tres medios (los que cubren el narcotráfico), en los que se puede apreciar con total claridad la utilización de algunos de los factores complementarios de noticiabilidad señalados previamente:

-1) Proceso:

-Ortiz Pinchetti, Francisco. "Secuestros, asaltos, homicidios, linchamientos y descrédito de los cuerpos de seguridad" [en línea]. Proceso, 6 de abril de 1996:

“...las modificaciones constitucionales aprobadas por el Senado de la República, y que analizan los diputados, van más allá del combate a la delincuencia: son leyes de control social, encaminadas a reprimir el malestar ciudadano provocado por las políticas económicas aplicadas por el gobierno actual...".

-Martín Morita, Cancino et al. "En su prisa por vender un avión implicado en presuntos ilícitos, atrae la atención de EU" [en línea]. Proceso, 2 de enero de 1999.

"Al armar el rompecabezas de las transacciones financieras de Raúl Salinas de Gortari — con el fin de probar que los fondos proceden del narcotráfico-, la policía suiza descubrió que en realidad las piezas encajan en una trama de mayor alcance, que incrimina a todo el grupo en el poder del sexenio pasado, en particular a la familia presidencial...".

-Ravelo, Ricardo. "La infiltración, desde Zedillo" [en línea]. Proceso, 22 de octubre de 2006:

"En el sexenio pasado, el gobierno de Ernesto Zedillo concibió un plan para negociar con el cártel de Juárez. De acuerdo con documentos ministeriales en poder de Proceso, el general Jorge Mariano Maldonado fue comisionado por la 
Secretaría de la Defensa y la Presidencia de la República para establecer contacto con el jefe de esa organización, Amado Carrillo, a fin de lograr un acuerdo que incluyera beneficios mutuos...".

\section{-2) Contralínea:}

-Merino, Elda . "El marcado negro de las armas" [en línea]. Contralínea, $\mathrm{n}^{\mathrm{o}} 13$, junio de 2003:

"El tráfico de armas se ha convertido en un problema serio para las autoridades que están imposibilitadas para frenarlo. Los vacíos legales y la infiltración del crimen en las corporaciones policíacas ha permitido un incremento del tráfico ilegal de armas provenientes del extranjero, principalmente de origen norteamericano, de acuerdo con fuentes policíacas. Se estima que entre el 2001 y 2002 , entraron a territorio mexicano por la frontera norte de manera ilegal 24 mil armas de fuego cortas y largas; un millón 224 mil 718 municiones de diferente calibre hacia el centro y sur del país, como el Estado de México, Distrito Federal y Guerrero. Los principales compradores del armamento, son narcotraficantes, bandas de asaltantes y roba-coches, entre otros, algunos comandados por integrantes y ex integrantes de las distintas corporaciones policíacas y castrenses...".

-Reyez, José. "Sobrino de Arévalo Gardoqui, vinculado al cártel de Cali” [en línea]. Contralínea, 21 de junio de 2009:

“...(por) información de inteligencia se sabe que Agustín Romero Villa y Jorge Gustavo Arévalo Kessler están involucrados en el decomiso de 3 toneladas 750 kilogramos de cocaína, mismos que pretendían trasladar de Ecuador al aeropuerto de Toluca, Estado de México"...”.

-Reyez, José. "En gobierno panistas fortificó su imperio el Cártel de Tijuana" [en línea]. Contralínea, 25 de octubre de 2009:

“... a la sombra de los gobiernos panistas, en Baja California" afianzó su poder corrompiendo a una gran parte de la "élite de la inteligencia militar, funcionarios de alto rango de la PGR, de la procuraduría estatal y de las policías Federal de Caminos, Ministerial y municipal" 